\title{
Mass loss by a scalar charge in an expanding universe
}

\author{
Lior M. Burko \\ Department of Physics, University of Utah, Salt Lake City, Utah 84112 \\ and Theoretical Astrophysics, California Institute of Technology, Pasadena, California 91125 \\ Abraham I. Harte \\ Theoretical Astrophysics, California Institute of Technology, Pasadena, California 91125 \\ Eric Poisson \\ Department of Physics, University of Guelph, Guelph, Ontario, Canada N1G 2W1 \\ (Received 7 January 2002; published 23 May 2002)
}

\begin{abstract}
We study the phenomenon of mass loss by a scalar charge - a point particle that acts as a source for a noninteracting scalar field-in an expanding universe. The charge is placed on comoving world lines of two cosmological spacetimes: a de Sitter universe, and a spatially flat, matter-dominated universe. In both cases, we find that the particle's rest mass is not a constant, but that it changes in response to the emission of monopole scalar radiation by the particle. In de Sitter spacetime, the particle radiates all of its mass within a finite proper time. In the matter-dominated cosmology, this happens only if the charge of the particle is sufficiently large; for smaller charges the particle first loses some of its mass, but then regains it all eventually.
\end{abstract}

DOI: 10.1103/PhysRevD.65.124006

PACS number(s): 04.25.-g, 95.30.Cq, 98.80.-k

\section{INTRODUCTION AND SUMMARY}

In this paper we study the phenomenon of mass loss by a scalar charge in an expanding universe. By scalar charge we mean a pointlike particle carrying a charge $q$ that acts as a source for a massless scalar field $\Phi$. The theory describing the particle-field system is a close analogue to standard electromagnetic theory. It comes with a wave equation for the field, and equations of motion for the particle; both equations are linear in $\Phi$. The equations predict that the mass of the particle is not a constant, but that it changes dynamically as the particle moves in a curved spacetime [1]. This comes about because the four-force produced by the scalar field, which is proportional to the gradient of $\Phi$, is not orthogonal to the particle's four-velocity. Although such an unusual situation was identified in the past [2], it has attracted surprisingly little attention, and its consequences are well worth exploring.

The usual notion of a particle's rest mass is that it is an invariant quantity that always stays constant. Further, one normally assumes that all electrons, say, have the same mass, which is then a fundamental constant of nature. Indeed, the whole concept of an elementary particle tends to imply restmass universality, and rest-mass conservation. It is also usually taken for granted that the properties of elementary particles_-such as mass, charge, or spin-are independent of the cosmological parameters. While suggestions were made to link cosmology to the properties of elementary particles (notably the large-numbers hypothesis [3-7]), this idea has not gained much popularity.

We show here that such a link is unavoidable for scalar charges - a scalar particle at rest in an expanding universe possesses a changing mass whose dynamics is directly coupled to the cosmology. We display this connection for two spacetimes: de Sitter, and a spatially flat matterdominated cosmology. A de Sitter universe provides a rea- sonable description of the inflationary epoch of our own universe, and the flat matter-dominated universe adequately models our universe's present epoch (in the absence of dark energy). Thanks to the simplicity of these spacetimes, our mathematical treatment of the mass-change phenomenon is exact: our calculations involve no approximations. The phenomenon is not exclusive to the two spacetimes considered here: A similar effect occurs in flat $(1+1)$-dimensional and $(2+1)$-dimensional Minkowski spacetimes, and this is discussed in a separate paper [8]. The effect, however, does not occur in $(3+1)$-dimensional Minkowski spacetime, nor in the Kerr family of spacetimes [9].

For both cosmologies we calculate the changing mass of a scalar charge taken to be comoving with the cosmological fluid. We assume that the particle starts with a finite mass $m_{0}$ in the finite past, and we determine how the mass behaves thereafter; its behavior depends on $m_{0}$ and $q$, as well as the cosmological parameters. For our matter-dominated universe, we find that two types of behaviors are possible, depending on the size of $q$ relative to a combination of other parameters. In the first scenario $(q$ small $)$, the particle first loses a fraction of its mass, but it then regains it all eventually. In the second scenario ( $q$ large), the particle loses all of its mass within a finite proper time. For de Sitter spacetime, only the second scenario applies, and the particle loses mass at a constant rate.

The mechanism behind the mass-change phenomenon is easy to identify: the scalar charge emits monopole waves, and the energy carried off by the radiation is taken from the particle's rest mass. (As we show in the paper, this interpretation is precise for de Sitter spacetime, which admits a timelike Killing vector. It is not precise, but still loosely correct, in the case of our matter-dominated universe, which does not admit a timelike Killing vector.) The radiative process is fueled by the expansion of the universe (which provides the required dynamics), and its monopolar nature reflects the 
spherical symmetry of the problem. This implies that the mass-change phenomenon should not be expected for fields of higher spins: monopole waves could not be produced in such cases. Instead, the radiation would necessarily be associated with higher multipole moments, and it could not be produced by a changing rest mass. For example, the fourforce produced by an electromagnetic field is always orthogonal to the four-velocity, and the rest mass of an electric charge is always constant.

The classical framework adopted here does not allow us to predict what happens when a particle has radiated all of its mass. To avoid a runaway regime of negative rest masses, we assume that the scalar charge simply disappears when its mass drops to zero. This fix is imposed without a proper justification, but it seems reasonable, and it does not appear to violate any known law of physics. In this regard we remark that the theory adopted here to describe the particlefield system does not automatically enforce scalar charge conservation. We assume, for simplicity, that $q$ stays constant as long as the particle continues to exist, but that it jumps abruptly to zero when the particle has radiated all of its mass. It would be interesting to consider more sophisticated models in which the scalar charge, as well as the mass, would be dynamically changing. We shall not, however, pursue this here.

We note that our notion of a scalar charge-a pointlike particle acting as a source for a massless scalar field-is different from other models which also describe scalar particles. For example, Seidel and Suen [10] considered a soliton which is made of a massive, sourceless scalar field. (For the Seidel-Suen soliton it is important that the field be massive: first, the frequency of the soliton-star oscillations vanishes without the mass term, and the solution becomes unstable; second, from the studies of gravitational critical phenomena we know that a massless scalar field either disperses to infinity or collapses to a black hole [11].) The Seidel-Suen soliton is very different from our notion of a scalar charge. It would be interesting, however, to investigate how the Seidel-Suen soliton behaves in a cosmological spacetime.

We begin in Sec. II with the presentation of an action principle for the particle-field system, and a derivation of the equations of motion. In Sec. III we describe a class of cosmological spacetimes that includes the two cosmologies of interest to us. In Sec. IV we calculate the retarded Green's function for a scalar field living in these two spacetimes. An alternative derivation, specific to de Sitter spacetime, is described in the Appendix. In Sec. V we compute the field of a scalar charge at rest in the two spacetimes, and show that the particle radiates monopole waves. The mass loss is computed in Sec. VI. Finally, Sec. VII contains a discussion of energy conservation in de Sitter spacetime, and an examination of the implications of the mass-loss effect on the abundance of scalar charges in our own universe. We also consider how our purely classical treatment might be related to a fundamental quantum theory of radiating scalar charges. Throughout the paper, except when stated otherwise, we use geometrized units in which $c=G=1$.

\section{ACTION PRINCIPLE AND EQUATIONS OF MOTION}

A particle of bare mass $m_{0}$ and scalar charge $q$ moves on a world line $z^{\alpha}(\lambda)$ in a curved spacetime with metric $g_{\alpha \beta}$; the world line is monotonically parametrized by $\lambda$. The particle creates a scalar field $\Phi$, and the dynamics of the particle-field system is described by the action principle

$$
\begin{aligned}
S= & \int\left\{-\frac{1}{8 \pi} g^{\alpha \beta} \Phi_{, \alpha} \Phi_{, \beta}-\int\left(m_{0}-q \Phi\right)\right. \\
& \left.\times \sqrt{-g_{\alpha \beta} \dot{z}^{\alpha} \dot{z}^{\beta}} \frac{\delta_{4}(x-z(\lambda))}{\sqrt{-g}} d \lambda\right\} \sqrt{-g} d^{4} x,
\end{aligned}
$$

where $\Phi_{, \alpha} \equiv \partial \Phi / \partial x^{\alpha}, \dot{z}^{\alpha} \equiv d z^{\alpha} / d \lambda, g$ is the metric determinant, and $\delta_{4}(x-z)$ is a four-dimensional Dirac distribution which satisfies $\int \delta_{4}(x-z) d^{4} x=1$ if $z^{\alpha}$ is within the domain of integration. We note that the action $S$ is invariant under a reparametrization of the world line. After variation, it is convenient to set $\lambda$ equal to the particle's proper time $\tau$, which is obtained by integrating $d \tau=\sqrt{-g_{\alpha \beta} \dot{z}^{\alpha} \dot{z}^{\beta}} d \lambda$. We also note that the metric $g_{\alpha \beta}$ does not participate in the dynamics; it is a prescribed tensor field in spacetime.

Variation of the action with respect to $\Phi$ produces a linear wave equation for the field,

$$
g^{\alpha \beta} \nabla_{\alpha} \nabla_{\beta} \Phi=-4 \pi \mu,
$$

where

$$
\mu(x)=q \int \frac{\delta_{4}(x-z(\tau))}{\sqrt{-g}} d \tau
$$

is the scalar charge density. Variation of the action with respect to $z^{\alpha}(\lambda)$ produces equations of the motion for the particle,

$$
m(\tau) \frac{D u^{\alpha}}{d \tau}=q\left(g^{\alpha \beta}+u^{\alpha} u^{\beta}\right) \Phi_{, \beta},
$$

where $u^{\alpha}=d z^{\alpha} / d \tau$ is the four-velocity, $D u^{\alpha} / d \tau \equiv d u^{\alpha} / d \tau$ $+\Gamma_{\beta \gamma}^{\alpha} u^{\beta} u^{\gamma}$ the covariant acceleration, and

$$
m(\tau)=m_{0}-q \Phi
$$

the dynamical mass of the particle. This last equation is equivalent to the differential statement

$$
\frac{d m}{d \tau}=-q \Phi_{, \alpha} u^{\alpha}
$$

which is independent of the bare mass $m_{0}$.

We note that Eqs. (2.2)-(2.4) and (2.6) also follow from an action principle proposed by Quinn [1]: 


$$
\begin{aligned}
S^{\prime}= & \int\left\{-\frac{1}{8 \pi} g^{\alpha \beta} \Phi_{, \alpha} \Phi_{, \beta}+\int\left[\frac{1}{2} m(\tau) g_{\alpha \beta} u^{\alpha} u^{\beta}\right.\right. \\
& \left.+q \Phi] \frac{\delta_{4}(x-z(\tau))}{\sqrt{-g}} d \tau\right\} \sqrt{-g} d^{4} x .
\end{aligned}
$$

This action involves only the dynamical mass $m(\tau)$, but it is not invariant under a reparametrization of the world line.

The actions $S$ and $S^{\prime}$ both produce a dynamically changing mass for a scalar charge. It is possible to construct an action that produces a constant mass. For example, replacing $\left(m_{0}-q \Phi\right)$ in Eq. (2.1) by $m_{0} \exp \left(-q \Phi / m_{0}\right)$ gives rise to the equations of motion $m_{0} D u^{\alpha} / d \tau=q\left(g^{\alpha \beta}+u^{\alpha} u^{\beta}\right) \Phi_{, \beta}$. The price to pay, however, is high, as Eq. (2.2) must now be replaced by the nonlinear wave equation $g^{\alpha \beta} \nabla_{\alpha} \nabla_{\beta} \Phi=$ $-4 \pi \mu \exp \left(-q \Phi / m_{0}\right)$.

As they stand, Eqs. (2.4)-(2.6) have only formal validity because the field $\Phi$ derived from Eqs. (2.2) and (2.3) is singular on the world line. Quinn [1] has shown that the singular part of the field does not affect the motion of the particle, which is then governed entirely by the smooth (or tail) part of the field. Quinn has thus produced regularized versions of the particle's equations of motion:

$$
\begin{aligned}
m \frac{D u^{\alpha}}{d \tau}= & \frac{1}{6} q^{2}\left(R_{\beta}^{\alpha} u^{\beta}+u^{\alpha} R_{\beta \gamma} u^{\beta} u^{\gamma}\right)+q^{2}\left(g^{\alpha \beta}\right. \\
& \left.+u^{\alpha} u^{\beta}\right) \int_{-\infty}^{\tau_{-}} G_{, \beta}\left(\tau, \tau^{\prime}\right) d \tau^{\prime}
\end{aligned}
$$

and

$$
\frac{d m}{d \tau}=-\frac{1}{12} q^{2} R-q^{2} u^{\alpha} \int_{-\infty}^{\tau_{-}} G_{, \alpha}\left(\tau, \tau^{\prime}\right) d \tau^{\prime} .
$$

Here, $R_{\alpha \beta}$ is the spacetime's Ricci tensor, and $R$ is the Ricci scalar. The quantity $G\left(x, x^{\prime}\right)$ appearing inside the integrals is the retarded Green's function associated with the scalar wave equation (2.2); it satisfies

$$
g^{\alpha \beta} \nabla_{\alpha} \nabla_{\beta} G\left(x, x^{\prime}\right)=-4 \pi \frac{\delta_{4}\left(x-x^{\prime}\right)}{\sqrt{-g}},
$$

where $x$ is identified with $z(\tau)$, the current position of the particle, while $x^{\prime}$ is identified with $z\left(\tau^{\prime}\right)$, the particle's past position. The integrals extend over the entire past world line of the particle, from $\tau^{\prime}=-\infty$ to (almost) the current time, $\tau^{\prime}=\tau^{-} \equiv \tau-\epsilon$, where $\epsilon$ is infinitesimally positive [1]. The integration is cut short to avoid the singular behavior of the Green's function as $x^{\prime}$ approaches $x$; it involves only the smooth part of the Green's function, which is often referred to as its "tail part." In Eqs. (2.8) and (2.9), the four-velocity $u^{\alpha}$ is evaluated at the current time $\tau$, and the Green's function is differentiated with respect to $x$ before making the identification $x=z(\tau)$.

We will evaluate and solve Eq. (2.9) for the dynamical mass of a scalar charge at rest in an expanding universe-in this case the right-hand side of Eq. (2.8) vanishes and the particle follows a geodesic of the spacetime. We will see that solving Eq. (2.9) produces a regularized version of Eq. (2.5).

\section{COSMOLOGICAL SPACETIMES}

For simplicity we consider spatially-flat cosmologies, and write the metric as

$$
d s^{2}=a^{2}(\eta)\left(-d \eta^{2}+d x^{2}+d y^{2}+d z^{2}\right),
$$

in terms of a conformal time $\eta$. The spacetime is filled with a homogeneous fluid of density $\rho$, pressure $p$, and fourvelocity

$$
u^{\alpha}=\frac{d x^{\alpha}}{d \tau}=\left(a^{-1}, 0,0,0\right)
$$

where $\tau$ is proper time for comoving observers, related to the conformal time by $d \tau=a(\eta) d \eta$. The behavior of the scale factor $a(\eta)$ is governed by an energy-conservation equation, $\left(a^{3} \rho\right)^{\prime}+p\left(a^{3}\right)^{\prime}=0$, in which a prime indicates differentiation with respect to $\eta$, and Raychaudhuri's equation, $a^{\prime 2}$ $=(8 \pi / 3) \rho a^{4}$.

We shall consider power-law cosmologies, for which the scale factor takes the simple form

$$
a(\eta)=C \eta^{\alpha}
$$

where $C$ and $\alpha$ are constants. For such cosmologies, the density is given by

$$
\rho=\frac{3 \alpha^{2}}{8 \pi C^{2}} \frac{1}{\eta^{2 \alpha+2}},
$$

the pressure by

$$
p=\frac{2-\alpha}{3 \alpha} \rho
$$

and the Ricci scalar by

$$
R=\frac{6 \alpha(\alpha-1)}{C^{2}} \frac{1}{\eta^{2 \alpha+2}}
$$

Two special cases, which constitute an equivalence class in a sense to be described below, will be of interest to us. The first is a matter-dominated cosmology characterized by $\alpha$ $=2$, which produces a vanishing pressure. The second is a de Sitter cosmology characterized by $\alpha=-1$, which produces a constant density and a pressure $p=-\rho$. In Table I we summarize the properties of these cosmologies.

The scalar wave equation (2.2) can be simplified if we introduce the auxiliary field variable $\psi$ defined by

$$
\Phi(\eta, x)=\frac{1}{a(\eta)} \psi(\eta, x),
$$

in which $\boldsymbol{x}=(x, y, z)$ represents the spatial coordinates. This new variable satisfies 
TABLE I. The cosmological models considered in this paper. In both cases the scale factor is given by $a(\eta)=C \eta^{\alpha}$, and proper time is defined by $d \tau=a(\eta) d \eta$. For both cosmologies, the table displays the parameter $\alpha$, the scale factor (in terms of both $\eta$ and $\tau$ ), the relations $\tau(\eta)$ and $\eta(\tau)$, the density $\rho(\eta)$, the pressure-todensity ratio, and the Ricci scalar $R(\eta)$. In the spatially flat, matterdominated cosmology, both $C$ and $\eta$ are positive, and the universe is expanding. In the de Sitter cosmology, both $C$ and $\eta$ are negative, and $a(\eta)=|C| /(-\eta)$ also describes an expanding universe. (The maximally extended de Sitter spacetime also includes a preceding contracting phase which we do not consider here.)

\begin{tabular}{lcc}
\hline \hline Cosmology & Matter dominated & de Sitter \\
\hline$\alpha$ & 2 & -1 \\
$a(\eta)$ & $C \eta^{2}$ & $|C| /(-\eta)$ \\
$a(\tau)$ & $C(3 \tau / C)^{2 / 3}$ & $|C| e^{\tau /|C|}$ \\
$\tau(\eta)$ & $C \eta^{3} / 3$ & $-|C| \ln (-\eta)$ \\
$\eta(\tau)$ & $(3 \tau / C)^{1 / 3}$ & $-e^{-\tau / C \mid}$ \\
$\rho$ & $3 /\left(2 \pi C^{2} \eta^{6}\right)$ & $3 /\left(8 \pi C^{2}\right)$ \\
$p / \rho$ & 0 & -1 \\
$R$ & $12 /\left(C^{2} \eta^{6}\right)$ & $12 / C^{2}$ \\
\hline \hline
\end{tabular}

$$
\left[-\frac{\partial^{2}}{\partial \eta^{2}}+\nabla^{2}+\frac{\alpha(\alpha-1)}{\eta^{2}}\right] \psi=-4 \pi a^{3} \mu,
$$

where $\nabla^{2}=\partial^{2} / \partial x^{2}+\partial^{2} / \partial y^{2}+\partial^{2} / \partial z^{2}$ is the flat-space Laplacian operator. On the basis of Eq. (3.8) we can state the following interesting property: Two differing cosmological models with identical values for $\beta \equiv \frac{1}{2} \alpha(\alpha-1)$ will produce identical reduced fields $\psi$, provided that the source term $a^{3} \mu$ is the same in both cases, and that $\psi$ starts with the same initial conditions. In this specific sense, we may say that two cosmological models with equal $\beta$ are "equivalent." For a prescribed value of $\beta$, the two equivalent cosmological models will be characterized by $\alpha=\alpha_{+}$, where

$$
\alpha_{ \pm}=\frac{1}{2} \pm \frac{1}{2} \sqrt{1+8 \beta}
$$

Notice in particular that if $\beta=1$, then $\alpha_{+}=2$ and $\alpha_{-}=-1$, and we obtain the cosmological models summarized in Table I. These models are therefore equivalent in the sense adopted here.

\section{GREEN'S FUNCTION}

In order to find solutions to Eq. (2.10), we factorize the Green's function according to

$$
G\left(x, x^{\prime}\right)=\frac{1}{a(\eta) a\left(\eta^{\prime}\right)} g\left(x, x^{\prime}\right),
$$

which produces a reduced version of Green's equation,

$$
\left(-\frac{\partial^{2}}{\partial \eta^{2}}+\nabla^{2}+\frac{2 \beta}{\eta^{2}}\right) g\left(x, x^{\prime}\right)=-4 \pi \delta\left(\eta-\eta^{\prime}\right) \delta_{3}\left(\boldsymbol{x}-\boldsymbol{x}^{\prime}\right)
$$

In the following two subsections we shall solve Eq. (4.2) using two different methods: the first relies on Hadamard's theory, and the second is based on a mode decomposition of Green's equation. An alternative derivation, specific to de Sitter spacetime, is described in the Appendix.

\section{A. Solution by Hadamard ansatz}

For $\beta=0$ (flat spacetime), the retarded solution to Eq. (4.2) is

$$
g^{\text {flat }}\left(x, x^{\prime}\right)=\frac{\delta(u)}{\left|\boldsymbol{x}-\boldsymbol{x}^{\prime}\right|},
$$

where $u=\eta-\eta^{\prime}-\left|\boldsymbol{x}-\boldsymbol{x}^{\prime}\right|$ is retarded time; we see that the flat-spacetime Green's function has support only on the past light cone of the field point $x$. Relying on Hadamard's general theory $[12,13]$, we expect that $g\left(x, x^{\prime}\right)$ will have support inside the light cone as well, and we write it as

$$
g\left(x, x^{\prime}\right)=g^{\text {flat }}\left(x, x^{\prime}\right)+B\left(x, x^{\prime}\right) \theta(u),
$$

where $\theta(u)$ is the Heaviside step function and $B\left(x, x^{\prime}\right)$ a two-point function to be determined, but which is known to be smooth when $u=0$. [Because the right-hand side of Eq. (4.2) is nothing more than a flat-spacetime $\delta$ function, there is no need to modify the $\delta(u)$ part of the Green's function: it is simply equal to the flat-spacetime result. This assumption is justified by the following.] Substituting Eq. (4.4) into Eq. (4.2), we find that $g^{\text {flat }}$ takes care of the four-dimensional $\delta$ function, and that the remainder vanishes as a distribution if $B$ satisfies

$$
(\square+V) B=0
$$

and

$$
B_{, \alpha}\left(x-x^{\prime}\right)^{\alpha}+B-\frac{1}{2} V=\mathcal{O},
$$

where $\square=-\partial^{2} / \partial \eta^{2}+\nabla^{2} \quad$ is the flat-spacetime d'Alembertian operator and $V(x) \equiv 2 \beta / \eta^{2}$. The right-hand side of Eq. (4.6) is a priori arbitrary, but it must vanish when $u=0$; it is also constrained by the fact that a simultaneous solution to both Eqs. (4.5) and (4.6) must exist. As a consequence of Eq. (4.6) and the fact that $B$ is smooth at $u=0$, we establish the coincidence limit

$$
\lim _{x \rightarrow x^{\prime}} B\left(x, x^{\prime}\right)=\frac{1}{2} V
$$

This boundary condition allows us to solve Eqs. (4.5) and (4.6) uniquely.

For simplicity we shall set the right-hand side of Eq. (4.6) to zero. As we shall see, this will produce the condition $\beta$ $=1$, and we shall therefore be restricted to the cosmological models summarized in Table I.

Let $x$ and $x^{\prime}$ be fixed points in spacetime, and let the relations $x^{\prime \prime} \alpha=x^{\prime} \alpha+\left(\eta^{\prime \prime}-\eta^{\prime}\right) n^{\alpha}$ describe a straight line going from $x^{\prime}$ to $x$ as the parameter $\eta^{\prime \prime}$ ranges from $\eta^{\prime}$ to $\eta$; 
the tangent vector $n^{\alpha}=d x^{\alpha} / d \eta^{\prime \prime}$ is normalized so that $n^{\eta}$ $=1$. Rewrite Eq. (4.6) in terms of the variable $x^{\prime \prime}$ and note that the derivative of $B$ in the direction of $\left(x^{\prime \prime}-x^{\prime}\right)^{\alpha}$ is equal to $\eta^{\prime \prime} d B / d \eta^{\prime \prime}$. Equation (4.6) can therefore be re-expressed as

$$
\eta^{\prime \prime} \frac{d B}{d \eta^{\prime \prime}}+B=\frac{1}{2} V\left(\eta^{\prime \prime}\right)
$$

and this can be straightforwardly integrated. The solution that satisfies Eq. (4.7) is

$$
B\left(x, x^{\prime}\right)=\frac{1}{2\left(\eta-\eta^{\prime}\right)} \int_{\eta^{\prime}}^{\eta} V\left(\eta^{\prime \prime}\right) d \eta^{\prime \prime}
$$

With $V=2 \beta / \eta^{2}$ we have that $B\left(x, x^{\prime}\right)=\beta /\left(\eta \eta^{\prime}\right)$.

Thus far we have generated a solution to Eq. (4.6) only. (Recall that we have set $\mathcal{O}=0$ in this equation.) We must now check that this is also a solution to Eq. (4.5). Because both $V$ and $B$ are proportional to $\beta$, it is easy to see that this produces a constraint on the value of $\beta$. As we have indicated previously, $B\left(x, x^{\prime}\right)=\beta /\left(\eta \eta^{\prime}\right)$ is a solution to both Eqs. (4.5) and (4.6) if and only if $\beta=1$.

Our conclusion is that for $\beta=1$ (which implies either $\alpha$ $=2$ or $\alpha=-1$ ), the retarded solution to Eq. (4.2) is

$$
g\left(x, x^{\prime}\right)=\frac{\delta\left(\eta-\eta^{\prime}-\left|\boldsymbol{x}-\boldsymbol{x}^{\prime}\right|\right)}{\left|\boldsymbol{x}-\boldsymbol{x}^{\prime}\right|}+\frac{\theta\left(\eta-\eta^{\prime}-\left|\boldsymbol{x}-\boldsymbol{x}^{\prime}\right|\right)}{\eta \eta^{\prime}} .
$$

The cosmological models summarized in Table I are equivalent in the sense used before, but also in the sense that they come with the same reduced Green's function $g\left(x, x^{\prime}\right)$. Because the scale factors are different, however, the actual retarded Green's function $G\left(x, x^{\prime}\right)$, given by Eq. (4.1), takes a distinct form in each spacetime.

\section{B. Solution by mode sum}

The method used in the preceding subsection to generate the retarded Green's function was limited to the case $\beta=1$. Here we describe an alternative method which could, if desired, be applied to any value of $\beta$. Here we set $\beta=\frac{1}{2} l(l$ +1 ), and we notice that this covers cosmological models characterized by either $\alpha=l+1$ or $\alpha=-l$. We will consider the case $l=1$ in detail, and reproduce Eq. (4.10).

We expand the reduced Green's function $g\left(x, x^{\prime}\right)$ in terms of plane-wave solutions to Laplace's equation,

$$
g\left(x, x^{\prime}\right)=\frac{1}{(2 \pi)^{3}} \int \tilde{g}\left(\eta, \eta^{\prime} ; \boldsymbol{k}\right) e^{i \boldsymbol{k} \cdot\left(\boldsymbol{x}-\boldsymbol{x}^{\prime}\right)} d^{3} k,
$$

and we substitute this into Eq. (4.2). The result, after also Fourier transforming $\delta_{3}\left(\boldsymbol{x}-\boldsymbol{x}^{\prime}\right)$, is an ordinary differential equation for $\tilde{g}\left(\eta, \eta^{\prime} ; \boldsymbol{k}\right)$ :

$$
\left[\frac{d^{2}}{d \eta^{2}}+k^{2}-\frac{l(l+1)}{\eta^{2}}\right] \tilde{g}=4 \pi \delta\left(\eta-\eta^{\prime}\right),
$$

where $k^{2}=\boldsymbol{k} \cdot \boldsymbol{k}$. To generate the retarded Green's function we set

$$
\tilde{g}\left(\eta, \eta^{\prime} ; \boldsymbol{k}\right)=\theta\left(\eta-\eta^{\prime}\right) \hat{g}\left(\eta, \eta^{\prime} ; k\right)
$$

in which we indicate that $\hat{g}$ depends only on the modulus of the vector $\boldsymbol{k}$. Substitution of Eq. (4.13) into Eq. (4.12) reveals that $\hat{g}$ must satisfy the homogeneous version of Eq. (4.12),

$$
\left[\frac{d^{2}}{d \eta^{2}}+k^{2}-\frac{l(l+1)}{\eta^{2}}\right] \hat{g}=0,
$$

together with the boundary conditions

$$
\hat{g}\left(\eta=\eta^{\prime} ; k\right)=0, \quad \frac{d \hat{g}}{d \eta}\left(\eta=\eta^{\prime} ; k\right)=4 \pi .
$$

Substitution of Eq. (4.13) into Eq. (4.11) and integration over the angular variables associated with $\boldsymbol{k}$ yields

$$
g\left(x, x^{\prime}\right)=\frac{\theta\left(\eta-\eta^{\prime}\right)}{2 \pi^{2} R} \int_{0}^{\infty} \hat{g}\left(\eta, \eta^{\prime} ; k\right) k \sin (k R) d k,
$$

where $R \equiv\left|\boldsymbol{x}-\boldsymbol{x}^{\prime}\right|$.

For $l=0$, the unique solution to Eqs. (4.14) and (4.15) is

$$
\hat{g}_{l=0}\left(\eta, \eta^{\prime} ; k\right)=\frac{4 \pi}{k} \sin (k \Delta \eta),
$$

where $\Delta \eta=\eta-\eta^{\prime}$. Substituting this into Eq. (4.16) returns the flat-spacetime Green's function of Eq. (4.3). To derive this we make use of the identity

$$
\frac{2}{\pi} \int_{0}^{\infty} \sin (\omega x) \sin \left(\omega x^{\prime}\right) d \omega=\delta\left(x-x^{\prime}\right)-\delta\left(x+x^{\prime}\right),
$$

and we note that the second $\delta$-function is eliminated by the step function $\theta(\Delta \eta)$ in Eq. (4.16).

If $l$ is an integer different from zero, Eq. (4.14) can be solved in terms of spherical Bessel functions. (If $l$ is not an integer, the equation can still be solved in terms of ordinary Bessel functions.) It is simpler, however, to generate solutions with the ladder operator $L_{l} \equiv-d / d \eta+(l+1) / \eta$. This works as follows. Suppose that we already have $\hat{g}_{l}$, a solution to Eq. (4.14) with a given value of $l$; then $\hat{g}_{l+1}=L_{l} \hat{g}_{l}$ is a solution to Eq. (4.14) with $l$ replaced by $l+1$. By repeated application of the ladder operator, a solution to Eq. (4.14) with any integer value of $l$ can be obtained from a seed solution $\hat{g}_{0}$.

We use this procedure to generate a solution $\hat{g}_{l=1}$ that satisfies the boundary conditions (4.15). For seed solutions we use the set $\{\sin (k \Delta \eta), \cos (k \Delta \eta)\}$. After application of $L_{0}$, we find that $\hat{g}_{l=1}$ must be given by a superposition of the linearly independent solutions $\cos (k \Delta \eta)-(k \eta)^{-1} \sin (k \Delta \eta)$ and $\sin (k \Delta \eta)+(k \eta)^{-1} \cos (k \Delta \eta)$. The coefficients are arbitrary 
functions of $\eta^{\prime}$, and after imposing Eqs. (4.15), we find that the appropriate combination is

$$
\begin{aligned}
\hat{g}_{l=1}\left(\eta, \eta^{\prime} ; k\right)= & \frac{4 \pi}{k}\left[\left(1+\frac{1}{k^{2} \eta \eta^{\prime}}\right) \sin (k \Delta \eta)\right. \\
& \left.-\frac{\Delta \eta}{k \eta \eta^{\prime}} \cos (k \Delta \eta)\right] .
\end{aligned}
$$

Substituting this into Eq. (4.16) and using Eq. (4.18) yields

$$
g_{l=1}\left(x, x^{\prime}\right)=\frac{\delta(\Delta \eta-R)}{R}+\frac{\theta(\Delta \eta)}{\eta \eta^{\prime}} I(\Delta \eta, R),
$$

where

$$
\begin{aligned}
I(\Delta \eta, R)= & \frac{2}{\pi R} \int_{0}^{\infty} \frac{\sin (k R)}{k^{2}}[\sin (k \Delta \eta) \\
& -(k \Delta \eta) \cos (k \Delta \eta)] d k .
\end{aligned}
$$

We evaluate this by integrating the first term by parts; this gives

$$
\begin{aligned}
I(\Delta \eta, R) & =\frac{2}{\pi} \int_{0}^{\infty} \frac{1}{k} \sin (k \Delta \eta) \cos (k R) d k \\
& =\theta(\Delta \eta-R) .
\end{aligned}
$$

We therefore arrive at

$$
g_{l=1}\left(x, x^{\prime}\right)=\frac{\delta(\Delta \eta-R)}{R}+\frac{\theta(\Delta \eta-R)}{\eta \eta^{\prime}},
$$

which agrees with Eq. (4.10).

The method described here could be extended to other values of $l$, but we shall not pursue this here.

\section{FIELD OF A STATIONARY CHARGE}

A particular solution to Eq. (2.2) is

$$
\Phi(x)=\int G\left(x, x^{\prime}\right) \mu\left(x^{\prime}\right) \sqrt{-g^{\prime}} d^{4} x^{\prime},
$$

where $G\left(x, x^{\prime}\right)$ is the retarded Green's function of Eq. (2.10) and $g^{\prime}$ is the determinant of the metric evaluated at $x^{\prime}$; the integration is over the entire spacetime manifold. In terms of the reduced variables $\psi(x)$ and $g\left(x, x^{\prime}\right)$-cf. Eqs. (3.7) and (4.1) —we have

$$
\psi(x)=\int g\left(x, x^{\prime}\right) a^{3}\left(\eta^{\prime}\right) \mu\left(x^{\prime}\right) d^{4} x^{\prime} .
$$

In this section we evaluate this for a stationary scalar charge.

The scalar charge density of a point particle is given by Eq. (2.3). For a particle at rest (comoving) in an expanding universe with metric (3.1), the four-velocity is given by Eq. (3.2), and Eq. (2.3) gives $a^{3}(\eta) \mu(\eta, x)=q \delta_{3}(\boldsymbol{x})$, if we choose $\boldsymbol{x}=0$ to represent the particle's position. (Because the spacetime is homogeneous, there is no loss of generality in this choice.) Substituting this into Eq. (5.2) gives

$$
\psi(\eta, x)=\int q g\left(\eta ; \boldsymbol{x}, \eta^{\prime} ; 0\right) d \eta^{\prime},
$$

where the reduced Green's function is given by Eq. (4.10).

For a matter-dominated cosmology $(\alpha=2, \beta=1$ - see Table I), the integration starts at $\eta^{\prime}=0$ where the Green's function is singular, and the integral of Eq. (5.3) is logarithmically divergent. For a de Sitter cosmology $(\alpha=-1, \beta$ $=1$ ), the integration starts at $\eta^{\prime}=-\infty$ and the integral is also logarithmically divergent. To avoid this pathology, we assume that the scalar charge came into being in the finite past, and we let

$$
q \rightarrow q \theta\left(\eta-\eta_{0}\right)
$$

in Eq. (5.3), which becomes

$$
\psi(\eta, x)=q \int_{\eta_{0}}^{\eta} g\left(\eta ; x, \eta^{\prime} ; 0\right) d \eta^{\prime} .
$$

The replacement of Eq. (5.4) describes the sudden creation of a scalar charge at a time $\eta=\eta_{0} \neq\{0,-\infty\}$, and this constitutes a simple cure for the pathology of Eq. (5.3). It is also possible to let the charge adiabatically "switch on," but this would needlessly lead to more complicated expressions. We recall that there is no law of charge conservation in this theory: a scalar charge can be spontaneously created provided that a sufficient amount of energy is made available.

Integration of Eq. (5.5), with the reduced Green's function of Eq. (4.11), is elementary, and we obtain

$$
\psi(\eta, x)=\frac{q}{r} \theta\left(\eta-r-\eta_{0}\right)\left[1+\frac{r}{\eta} \ln \left(\frac{\eta-r}{\eta_{0}}\right)\right],
$$

where $r=|\boldsymbol{x}|$ is the coordinate distance from the origin. Equation (5.6) describes the reduced scalar field of a stationary charge at $\boldsymbol{x}=0$; the full scalar field is given by $\Phi(\eta, x)$ $=\psi(\eta, x) / a(\eta)$. The step function in Eq. (5.6) indicates that the moment of charge creation is registered at a time $\eta$ $=\eta_{0}+r$ by an observer at a distance $r$ from the charge: the information travels at the speed of light. Equation (5.6) implies that a stationary scalar charge in an expanding universe radiates monopole waves: While the reduced scalar field $\psi$ is stationary in the immediate vicinity of the charge, the reorganization of the field lines caused by the underlying spacetime curvature (which is dynamical) produces radiation. The expansion of the universe also participates directly in the dynamics of the scalar field; this is reflected by the presence of the scale factor in the relation $\Phi=\psi / a$.

The field of Eq. (5.6) is singular at $\boldsymbol{x}=0$, where the particle is located. We define a renormalized local field $\psi_{\text {ren }}$ by first removing the singular part of $\psi$ and then taking the limit $r \rightarrow 0$. This gives

$$
\psi_{\mathrm{ren}}(\eta)=\frac{q}{\eta} \theta\left(\eta-\eta_{0}\right) \ln \left(\eta / \eta_{0}\right)
$$


We also define $\Phi_{\text {ren }}(\eta) \equiv \psi_{\text {ren }}(\eta) / a(\eta)$; the physical significance of this quantity will be revealed in the next section.

\section{MASS LOSS}

In this section we evaluate Eq. (2.9) for a stationary scalar charge in the two cosmological spacetimes described in Table I. For this situation, the particle's four-velocity is given by Eq. (3.2), and the relevant (smooth) part of the Green's function is obtained from Eqs. (4.1) and (4.10):

$$
G_{\text {smooth }}\left(x, x^{\prime}\right)=\frac{1}{C^{2}} \frac{1}{\left(\eta \eta^{\prime}\right)^{\alpha+1}} .
$$

We recall that the scale factor is given by $a(\eta)=C \eta^{\alpha}$, where $C$ and $\alpha$ are constants; Eq. (6.1) is valid if and only if $\alpha$ is restricted to the values 2 (matter-dominated cosmology) and -1 (de Sitter cosmology). We recall also that for these cosmologies, the Ricci scalar is given by Eq. (3.6).

For a stationary particle, $u^{\alpha} G_{, \alpha}$ reduces to $a^{-1} \partial_{\eta} G$, and the proper-time integral of Eq. (2.9) can be expressed as an integral over $d \eta^{\prime}=a^{-1}\left(\eta^{\prime}\right) d \tau^{\prime}$. This yields

$\frac{d m}{d \eta}=-\frac{1}{12} q^{2} a(\eta) R(\eta)-q^{2} \int_{\eta_{0}}^{\eta} \partial_{\eta} G_{\text {smooth }}\left(\eta, \eta^{\prime}\right) a\left(\eta^{\prime}\right) d \eta^{\prime}$

for the rate of change of the particle's dynamical mass. Notice that we have incorporated the cutoff of Eq. (5.4) into this expression.

\section{A. Matter-dominated cosmology}

We now evaluate Eq. (6.2) for $\alpha=2$. Substitution of Eqs. (6.1) and (3.6) yields

$$
\frac{d m}{d \eta}=-\frac{q^{2}}{C \eta^{4}}\left[1-3 \ln \left(\eta / \eta_{0}\right)\right]
$$

and this can be immediately integrated:

$$
m(\eta)=m_{0}-\frac{q^{2}}{C \eta^{3}} \ln \left(\eta / \eta_{0}\right),
$$

where $m_{0} \equiv m\left(\eta_{0}\right)$. This result can be restated in terms of the renormalized local field of Eq. (5.7):

$$
m(\eta)=m_{0}-q \Phi_{\text {ren }}(\eta),
$$

which is analogous to Eq. (2.5).

In order to analyze Eq. (6.4) we express it as

$$
\frac{m(\eta)}{m_{0}}=1-\frac{c}{x^{3}} \ln (x) \equiv f(x ; c),
$$

in terms of the rescaled quantities

$$
x=\frac{\eta}{\eta_{0}}, \quad c=\frac{q^{2}}{C m_{0} \eta_{0}^{3}} .
$$

The function $f(x ; c)$ is defined in the interval $1 \leqslant x<\infty$. It initially decreases from $f(1 ; c)=1$ as $x$ increases, and reaches its minimum value $f_{\min }=1-c /(3 e)$ at $x=e^{1 / 3}$ $\simeq 1.3956$. Then $f(x ; c)$ starts to increase, and it is eventually restored to its original value, $f(x \rightarrow \infty ; c) \rightarrow 1$. For $c \geqslant 3 e$ $\simeq 8.1548, f(x ; c)<0$ in an interval around $x=e^{1 / 3}$.

From these considerations, we conclude that for $q^{2}$ $<3 e \mathrm{Cm}_{0} \eta_{0}^{3}$, the particle first loses mass, but eventually regains all of it as $\eta \rightarrow \infty$. For $q^{2}>3 e C m_{0} \eta_{0}^{3}$, on the other hand, the particle radiates all of its mass in a time shorter than $\Delta \eta=\left(e^{1 / 3}-1\right) \eta_{0} \simeq 0.3956 \eta_{0}$; as was conjectured in Sec. I, this presumably signals the destruction of the scalar charge.

\section{B. de Sitter cosmology}

For $\alpha=-1, G_{\text {smooth }}\left(\eta, \eta^{\prime}\right)$ is a constant, and the integral term of Eq. (6.2) contributes nothing to the mass loss. Instead, this comes entirely from the Ricci-scalar term, and we find

$$
\frac{d m}{d \tau}=-\frac{q^{2}}{C^{2}}
$$

In terms of proper time $\tau$, mass is being lost at a constant rate. This result can also be expressed as

$$
m(\tau)=m_{0}-\frac{q^{2}}{C^{2}}\left(\tau-\tau_{0}\right),
$$

where $m_{0} \equiv m\left(\tau_{0}\right)$ is the initial mass of the scalar charge, and the proper time $\tau_{0}$ is related to the conformal time $\eta_{0}$. (The relevant relations between proper and conformal times are listed in Table I.) Here also we find that Eq. (6.9) can be simply restated in terms of the renormalized local field of Eq. (5.7):

$$
m(\tau)=m_{0}-q \Phi_{\text {ren }}(\tau)
$$

this again is analogous to Eq. (2.5).

Our conclusion here is that the scalar charge will radiate all of its mass, at a constant rate, within a proper time $\Delta \tau$ $=C^{2} m_{0} / q^{2}$. This again signals the destruction of the scalar particle.

\section{DISCUSSION: MASS LOSS IN de SITTER SPACETIME}

\section{A. Energy conservation}

The de Sitter spacetime possesses a maximal set of ten linearly independent Killing vectors, and each one can be associated with a global conservation law. The spatial symmetries of the problem immediately imply linear and angular momentum conservation, but the explicit time dependence does not permit a hasty dismissal of energy conservation. Here we show that energy is globally conserved: the energy radiated by a stationary scalar charge in de Sitter spacetime is exactly equal to the mass lost. We note that such an analy- 
sis cannot be adapted to the case of a matter-dominated cosmology, because this spacetime does not admit a timelike Killing vector.

Let $T^{\alpha \beta}$ be the stress-energy tensor of the particle-field system, and let $\xi^{\alpha}$ denote the timelike Killing vector of de Sitter spacetime. By virtue of the equations $T_{; \beta}{ }^{\alpha \beta}=0$ and $\xi_{\alpha ; \beta}+\xi_{\beta ; \alpha}=0$ we find that the vector $j^{\alpha}=-T_{\beta}^{\alpha} \xi^{\beta}$ is divergence free. By integrating $j_{; \alpha}^{\alpha}=0$ over a bounded fourdimensional volume $V$ and using Gauss' theorem, we obtain the conservation statement

$$
E \equiv-\oint_{\partial V} T_{\beta}^{\alpha} \xi^{\beta} d \Sigma_{\alpha}=0,
$$

where $\partial V$ is the volume's boundary, and $d \Sigma_{\alpha}$ is an outwarddirected surface element on $\partial V$; if $\left(y^{1}, y^{2}, y^{3}\right)$ are coordinates intrinsic to $\partial V$, then

$$
d \Sigma_{\mu}=\varepsilon_{\mu \alpha \beta \gamma}\left(\partial x^{\alpha} / \partial y^{1}\right)\left(\partial x^{\beta} / \partial y^{2}\right)\left(\partial x^{\gamma} / \partial y^{3}\right) d^{3} y,
$$

where $\varepsilon_{\mu \alpha \beta \gamma}$ is the totally antisymmetric Levi-Civita tensor.

The total stress-energy tensor $T^{\alpha \beta}$ is obtained by varying the action of Eq. (2.1) with respect to the metric. We express it as

$$
T^{\alpha \beta}=T_{\text {field }}^{\alpha \beta}+T_{\text {particle }}^{\alpha \beta},
$$

where

$$
T_{\alpha \beta}^{\mathrm{field}}=\frac{1}{4 \pi}\left(\Phi_{, \alpha} \Phi_{, \beta}-\frac{1}{2} g_{\alpha \beta} \Phi^{, \mu} \Phi_{, \mu}\right)
$$

is the stress-energy tensor of the scalar field, and

$$
T_{\text {particle }}^{\alpha \beta}=\int m(\tau) u^{\alpha}(\tau) u^{\beta}(\tau) \frac{\delta(x-z(\tau))}{\sqrt{-g}} d \tau
$$

is the stress-energy tensor of the particle.

We want to evaluate Eq. (7.1) when $\partial V$ consists of a cylindrical "tube" $B$ surrounding the particle's world line, closed off by "caps" $C_{1}$ and $C_{2}$ (each of constant time) at both ends. It would be inconvenient to carry out this computation in the cosmological coordinates $(\eta, x, y, z)$, because in these coordinates the metric is explicitly time dependent, and the Killing symmetry is poorly represented. We therefore prefer to use static coordinates $\left(t, r^{*}, \theta, \phi\right)$, in which the metric is explicitly time independent. The transformation is

$$
\begin{aligned}
\eta= & -\cosh \left(\kappa r^{*}\right) e^{-\kappa t}, \\
(x, y, z)= & \sinh \left(\kappa r^{*}\right) e^{-\kappa t} \\
& \times(\sin \theta \cos \phi, \sin \theta \sin \phi, \cos \theta),
\end{aligned}
$$

where $\kappa \equiv 1 /|C|$, and in the new coordinates, the de Sitter metric takes the form

$$
d s^{2}=\frac{-d t^{2}+d r^{* 2}}{\cosh ^{2}\left(\kappa r^{*}\right)}+\kappa^{-2} \tanh ^{2}\left(\kappa r^{*}\right)\left(d \theta^{2}+\sin ^{2} \theta d \phi^{2}\right) .
$$

In these coordinates, $\xi^{\alpha}=\delta^{\alpha}{ }_{t}$, and the Green's function of Eqs. (4.1) and (4.10) is

$$
G\left(x, x^{\prime}\right)=\kappa \operatorname{coth}\left(\kappa r^{*}\right) \delta\left(t-t^{\prime}-r^{*}\right)+\kappa^{2} \theta\left(t-t^{\prime}-r^{*}\right)
$$

if the source point is at the spatial origin of the coordinate system. The field of a stationary charge at that position is given by

$$
\Phi\left(t, r^{*}\right)=q \kappa \theta\left(t-r^{*}-t_{0}\right)\left[\operatorname{coth}\left(\kappa r^{*}\right)+\kappa\left(t-r^{*}-t_{0}\right)\right],
$$

where $t_{0}$ denotes the time at which the scalar charge came into being. Because $t$ is proper time for an observer at $r^{*}$ $=0$, the dynamical mass of the scalar particle can be expressed as

$$
m(t)=m_{0}-q^{2} \kappa^{2}\left(t-t_{0}\right),
$$

where $m_{0}$ is the particle's mass at the time $t_{0}$; this follows directly from Eq. (6.9).

We choose the closed hypersurface $\partial V$ to be the union of a three-cylinder $B$ described by $\left(r^{*}=R, t_{1}<t<t_{2}\right)$, a spherical ball $C_{1}$ described by $\left(t=t_{1}, 0<r^{*}<R\right)$, and another ball $C_{2}$ described by $\left(t=t_{2}, 0<r^{*}<R\right)$. After decomposing the total stress-energy tensor as in Eq. (7.2), we find that Eq. (7.1) becomes

$$
\begin{aligned}
E= & E_{\text {field }}[B]+E_{\text {field }}\left[C_{2}\right]-E_{\text {field }}\left[C_{1}\right]+E_{\text {particle }}[B] \\
& +E_{\text {particle }}\left[C_{2}\right]-E_{\text {particle }}\left[C_{1}\right]=0,
\end{aligned}
$$

where, for example, $E_{\text {field }}[B]=-\int_{B} T_{\text {field } \beta}^{\alpha} \xi^{\beta} d \Sigma_{\alpha}$. The minus signs in front of the terms associated with $C_{1}$ reflect the fact that the future-directed surface element on $C_{1}$ points within the region $V$, and therefore against the outwarddirected surface element on $\partial V$.

Omitting all calculational details, we now present our results for the various quantities appearing in Eq. (7.10). First, we find that

$$
\begin{aligned}
E_{\text {field }}\left[C_{2}\right]= & E_{\text {field }}\left[C_{1}\right]=\frac{1}{2} q^{2} \kappa^{2} \int_{0}^{R}\left[\tanh ^{2}\left(\kappa r^{*}\right)\right. \\
& \left.+\operatorname{coth}^{2}\left(\kappa r^{*}\right)\right] d r^{*} .
\end{aligned}
$$

This represents the field energy enclosed within a sphere of coordinate radius $R$ centered at the origin. Because the field configuration is singular at $r^{*}=0$, both $E_{\text {field }}\left[C_{2}\right]$ and $E_{\text {field }}\left[C_{1}\right]$ are formally infinite. A proper derivation of global energy conservation should therefore involve a regularization procedure for the field's energy. Fortunately, equality of $E_{\text {field }}\left[C_{2}\right]$ and $E_{\text {field }}\left[C_{1}\right]$ ensures that independently of the details of the regularization procedure, these quantities cancel each other out on the right-hand side of Eq. (7.10). (A 
technical requirement is that the regularization procedure must not violate the time-translational invariance of the field energy.) Second, we have

$$
E_{\text {field }}[B]=q^{2} \kappa^{2}\left(t_{2}-t_{1}\right),
$$

which represents the energy radiated by the particle during the time interval $t_{1}<t<t_{2}$ (notice that this is independent of $R$ ). Third,

$$
E_{\text {particle }}\left[C_{2}\right]=m\left(t_{2}\right), \quad E_{\text {particle }}\left[C_{1}\right]=m\left(t_{1}\right),
$$

and $E_{\text {particle }}[B]=0$ : the energy carried by the particle is equal to the current value of its mass.

Substituting Eqs. (7.12) and (7.13) into Eq. (7.10) yields

$$
E=q^{2} \kappa^{2}\left(t_{2}-t_{1}\right)+m\left(t_{2}\right)-m\left(t_{1}\right) .
$$

Using Eq. (7.9) reveals that energy is globally conserved: the change in the mass is exactly equal to the energy radiated, and $E=0$.

\section{B. Implications for this universe}

Can the mass-loss effect be used to explain the observed absence of scalar charges? We argue here that the answer is in the affirmative. We will calculate an upper bound on the total number of scalar charges that might still exist today, and show that it is very small, of the order of millions per galaxy. And we will calculate a lower bound on the mass of a scalar charge, and show that it is too large to permit the production of these particles in today's accelerators. We assume that scalar charges already existed at the onset of the inflationary epoch, and that no additional scalar charges were created since.

The following discussion must be preceded by an important disclaimer. The mass-loss phenomenon was investigated in Sec. VI within a framework in which the degrees of freedom associated with the charge and its scalar field were both described by classical physics. While we can hope that such a treatment may not be blatantly at odds with a proper quantum description, the following speculations regarding the fate of elementary scalar charges must be regarded as more conjecture than definite prediction. In this regard we can be encouraged by the fact that the classical treatment of electromagnetic radiation reaction is often not a bad approximation to the correct quantum description [14].

A fundamental quantum theory of radiating scalar charges might be based on the following considerations. The first ingredient would be a complex scalar field $\Phi_{\text {particle }}$ whose quantum excitations would give rise to massive particles that carry a scalar charge. The mass of the particles would be identified by locating the poles of the Feynman propagator in momentum space [15]. In the absence of radiative corrections, and in flat spacetime, these particles would have a mass that would stay constant, and this mass would directly correspond to the mass parameter of the field's Lagrangian. The physics of these elementary excitations might, however, be substantially different in the curved spacetime of an expanding universe. For example, Redmount [16] has shown that in the context of a real scalar field in de Sitter spacetime, the particle energies are not constant-they oscillate at late and early times - and do not correspond to the Lagrangian's mass parameter. Although Redmount's particles do not carry a scalar charge, his results clearly suggest that the quantum physics of a scalar charge in an expanding universe, even in the absence of radiative corrections, might be considerably richer than the classical description provided in this paper.

The second ingredient involved in a fundamental theory of radiating scalar charges would be another scalar field $\Phi_{\text {radiation }}$ coupled to the first to allow the scalar particles to radiate. This new field would be real and massless, and it would generate the radiative corrections that have so far been missing in our description. These would modify the energies of the scalar charges with respect to the (already complicated) free-field behavior. While this fully quantum description of a radiating scalar charge would undoubtedly be richer and more interesting than the classical treatment provided in this paper, we can hope that our classical considerations will not lead us too far astray - they should indeed provide us with a useful approximation. In this spirit we shall pursue our speculations regarding the fate of scalar charges in our own inflationary universe.

For concreteness we assume that the time scale associated with the inflationary epoch is $t_{c} \equiv|C| \sim 10^{-34} \mathrm{~s}$. This produces a distance scale $r_{c} \equiv c t_{c} \sim 3 \times 10^{-24} \mathrm{~cm}$, a density scale $\rho_{c} \equiv 3 /\left(8 \pi G t_{c}^{2}\right) \sim 2 \times 10^{74} \mathrm{~g} / \mathrm{cm}^{3}$, and a mass scale $m_{c} \equiv 4 \pi \rho_{c} r_{c}^{3} / 3 \sim 2 \times 10^{4} \mathrm{~g}$. For the purpose of this discussion we reintroduce the speed of light $c$ and the gravitational constant $G$, which were both previously set equal to one.

We assume that a number $N$ of scalar charges, all of the same mass $m_{0}$, are created prior to the onset of inflation, and that during inflation, their mass decays according to Eq. (7.9). An upper bound on $N$ is obtained by noting that at the onset of inflation, $N m_{0}$ cannot exceed $m_{c}$, the total mass contained in (what will become) the observable universe. Thus, $N<m_{c} / m_{0}$.

Whether or not the scalar charges will survive the inflationary epoch depends on the relation between $m_{0}$ and its duration $\Delta t$ : if the particles are created heavy, and if inflation does not persist for too long, then every particle present initially will still be present after inflation. With our objective to produce an upper bound for $N$, we assume that the scalar charges do survive the inflationary epoch, and we use Eq. (7.9) to derive a lower bound on the initial mass $m_{0}$. After inserting the appropriate factors of $c$ and normalizing the (unknown) scalar charge $q$ to the electron's charge $e$, we obtain

$$
m_{0}>\frac{e^{2}}{c^{3} t_{c}}\left(\frac{q}{e}\right)^{2}\left(\frac{\Delta t}{t_{c}}\right)
$$

For an inflationary epoch that persists for approximately 60 $e$-folding times, we have that $m_{0}>5 \times 10^{-15}(q / e)^{2} \mathrm{~g}$. If q/e is of order unity, this mass is larger by 6 orders of magnitude than the energy currently available at particle accelerators.

Combining this lower bound on $m_{0}$ with our previous result for $N$, we find that $N<4 \times 10^{18}(e / q)^{2}$, so that the total number of scalar charges present today cannot exceed 
$10^{7}(e / q)^{2}$ per galaxy. So we see that unless $e / q$ is huge, $m_{0}$ is naturally extremely large, $N$ is naturally extremely small, and the prospect of observing a scalar charge today is extremely limited.

\section{ACKNOWLEDGMENTS}

Our result for the retarded Green's function of a scalar field in de Sitter spacetime, and the realization that scalar charges in de Sitter spacetime must have a variable mass, were arrived at independently by Amos Ori [17]. We thank him for discussions, as well as Karel Kuchar, Lee Lindblom, and Kip Thorne. L.M.B. wishes to thank the Technion Institute of Theoretical Physics for hospitality. E.P. was supported by the Natural Sciences and Engineering Research Council (Canada). A.I.H. was supported by SURF. L.M.B. was supported by NSF grants PHY-0099568 (Caltech) and PHY9734871 (University of Utah).

\section{APPENDIX: ALTERNATIVE DERIVATION OF THE SCALAR FIELD GREEN'S FUNCTION FOR de SITTER SPACETIME}

In this appendix we present an alternative derivation for the retarded Green's function of a scalar field in de Sitter spacetime.

First, we observe that because of the four-dimensional homogeneity of de Sitter spacetime, the Green's function can only be a function of the invariant distance between two points. Next, we adopt coordinates that make it easy for us to use this observation. Specifically, we write the de Sitter metric in the form

$$
d s^{2}=-d \tau^{2}+\kappa^{-2} \sinh ^{2}(\kappa \tau)\left(d r^{2}+\sinh ^{2} r d \Omega^{2}\right),
$$

where $\kappa=|C|^{-1}$ and $d \Omega^{2}$ is the standard metric on the unit two-sphere. In the coordinates of Eq. (A1), the spatial sections of de Sitter spacetime are open hyperboloids of constant (negative) curvature. This form of the metric can be obtained from the embedding relations

$$
\begin{aligned}
& x_{0}=\kappa^{-1} \sinh (\kappa \tau) \cosh r \\
& x_{1}=\kappa^{-1} \cosh (\kappa \tau) \\
& x_{2}=\kappa^{-1} \sinh (\kappa \tau) \sinh r \cos \theta \\
& x_{3}=\kappa^{-1} \sinh (\kappa \tau) \sinh r \sin \theta \cos \phi \\
& x_{4}=\kappa^{-1} \sinh (\kappa \tau) \sinh r \sin \theta \sin \phi,
\end{aligned}
$$

which describe de Sitter spacetime as the hypersurface $-x_{0}^{2}+x_{1}^{2}+x_{2}^{2}+x_{3}^{2}+x_{4}^{2}=\kappa^{-2}$ in a five-dimensional flat spacetime. The coordinate ranges are $-\infty<\tau<\infty, 0 \leqslant r$ $<\infty, 0 \leqslant \theta \leqslant \pi$, and $0 \leqslant \phi \leqslant 2 \pi$. Notice that these coordinates do not cover the entire de Sitter manifold. They go bad on $\tau=0$ and $r=\infty$, which correspond to two null rays originating from the origin. More importantly, $\tau=0$ is a coordinate singularity: all the points (with finite coordinate values) ( $\tau$ $=0, r, \theta, \phi)$ are the same physical point on the manifold [17]. This can most easily be seen by direct substitution in the coordinate transformation of Eq. (A2).

These coordinates are particularly convenient, because surfaces of constant geodesic distance from the origin are also surfaces of constant $\tau$. We put the source at the origin of the coordinates without loss of generality (because of the homogeneity of de Sitter spacetime). Next, we will show that the proper time along a geodesic between the points $P_{0}$ at $(0,0,0,0)$ and $P_{1}$ at $(\tau, r, \theta, \phi)$ is simply $\tau$ for all finite values of $(r, \theta, \phi)$. This is most elegantly accomplished by a method suggested to us by Ori [17]. From the previous result that all the points with $\tau=0$ (and with finite coordinate values) are the same point, $P_{0}$ may be represented by the coordinates $(0, r, \theta, \phi)$, from which a constant $(r, \theta, \phi)$ curve (which is a geodesic) may be extended to the point $P_{1}$ at $(\tau, r, \theta, \phi)$. Next, observe that the proper time along geodesics of constant $(r, \theta, \phi)$ is simply the coordinate time. It then follows that the geodesic distance between $P_{0}$ and $P_{1}$ is $\tau$. The retarded Green's function sourced at $(0,0,0,0)$ is thus a function only of the coordinate time for all evaluation points, that is $G(x, 0)=G(\tau, 0)=G(\tau)$.

Next, using the previous result that $G=G(\tau)$, we use Green's equation to obtain an ordinary differential equation for $G(\tau)$. Specifically, we find that

$$
\sinh ^{-3}(\kappa \tau) \partial_{\tau}\left[\sinh ^{3}(\kappa \tau) \partial_{\tau} G(\tau)\right]=4 \pi \frac{\delta_{4}(x)}{\sqrt{-g}} .
$$

Inside the source's light cone, the Green's function is a linear combination of two linearly independent solutions for the corresponding homogeneous equation. These two solutions are just

$$
\begin{aligned}
& G_{1}(\tau)=1, \\
& G_{2}(\tau)=\frac{\cosh (\kappa \tau)}{\sinh ^{2}(\kappa \tau)}+\frac{1}{2} \ln \frac{\cosh (\kappa \tau)-1}{\cosh (\kappa \tau)+1} .
\end{aligned}
$$

Hadamard's theory [12] requires that $G(\tau)=c_{1} G_{1}(\tau)$ $+c_{2} G_{2}(\tau)$ be an analytic function. However, $G_{2}$ is not analytic: approaching $\tau=0$ it behaves like $G_{2}(\tau)=1 /\left(2 \kappa^{2} \tau^{2}\right)$ $+\ln (\tau) / 2+O(1)$. Hence, $c_{2}=0$, and we conclude that the Green's function is a constant inside the source's light cone.

The coordinates (A1) are inconvenient for finding the value of that constant, because of their bad behavior on the light cone. Because the Green's function is a scalar, our conclusion that it is a constant inside the light cone is unchanged when we switch to other coordinates. As was discussed in Sec. IV, it may be directly observed from the form of Green's equation in conformal coordinates that the support on the 
source's light cone is identical to the corresponding flatspace support. Transforming to the static coordinates of Eq. (7.6),

$$
G(x, 0)=\kappa \operatorname{coth}\left(\kappa r^{*}\right) \delta\left(t-r^{*}\right)+K \theta\left(t-r^{*}\right),
$$

where $K$ is yet to be determined.
Substituting this into the homogeneous Green's equation in these coordinates,

$$
\left[\cosh ^{2}\left(\kappa r^{*}\right)\left(-\partial_{t}^{2}+\partial_{r^{*}}^{2}\right)+2 \kappa \operatorname{coth}\left(\kappa r^{*}\right) \partial_{r^{*}}\right] G\left(t, r^{*}\right)=0,
$$

fixes $K=\kappa^{2}$. With this identification, Eq. (A5) is equivalent to the Green's function of Eqs. (4.1) and (4.10) adapted to de Sitter spacetime.
[1] T.C. Quinn, Phys. Rev. D 62, 064029 (2000).

[2] It is known, for example, that scalar theories of gravity do not automatically enforce the conservation of rest mass. See, for example, W.E. Thirring, Ann. Phys. (N.Y.) 16, 96 (1961) and references cited therein.

[3] A.S. Eddington, Proc. Cambridge Philos. Soc. 27, 15 (1931).

[4] P.A.M. Dirac, Nature (London) 139, 323 (1937).

[5] P.A.M. Dirac, Proc. R. Soc. London A165, 199 (1938).

[6] R.H. Dicke, Nature (London) 192, 440 (1961).

[7] B. Carter, in Confrontation of Cosmological Theories with $\mathrm{Ob}$ servational Data, edited by M. S. Longair (Reidel, Dordrecht, 1973).

[8] L.M. Burko, gr-qc/0201021.

[9] L.M. Burko and Y.T. Liu, Phys. Rev. D 64, 024006 (2001).
[10] E. Seidel and W.-M. Suen, Phys. Rev. Lett. 66, 1659 (1991).

[11] M.W. Choptuik, Phys. Rev. Lett. 70, 9 (1993).

[12] J. Hadamard, Lectures on Cauchy's Problem in Linear Partial Differential Equations (Yale University Press, New Haven, 1923).

[13] B.S. DeWitt and R.W. Brehme, Ann. Phys. (N.Y.) 9, 220 (1960).

[14] J. D. Jackson, Classical Electrodynamics, 2nd ed. (Wiley, New York, 1975), Chap. 17.

[15] M. E. Peskin and D. V. Schroeder, An Introduction to Quantum Field Theory (Addison-Wesley, Reading, MA, 1995).

[16] I.H. Redmount, Phys. Rev. D 40, 3343 (1989).

[17] A. Ori (personal communication). 